\title{
A new human chromogranin A (CgA) immunoradiometric assay involving monoclonal antibodies raised against the unprocessed central domain (145-245)
}

\author{
F Degorce', Y Goumon², L Jacquemart'1, C Vidaud'1, L Bellanger'1, D Pons-Anicet'1, P Seguin ${ }^{1}$, MH Metz-Boutigue ${ }^{2}$ \\ and D Aunis²
}

${ }^{1} \mathrm{CIS}$ biointernational, Division In Vitro Technologies, BP175, 30203 Bagnols-sur-Cèze, France; ' Institut National de la Santé et de la Recherche Médicale, INSERM U-338, Biologie de la Communication Cellulaire, 67084 Strasbourg, France

\begin{abstract}
Summary Chromogranin A ( $\mathrm{CgA})$, a major protein of chromaffin granules, has been described as a potential marker for neuroendocrine tumours. Because of an extensive proteolysis which leads to a large heterogeneity of circulating fragments, its presence in blood has been assessed in most cases either by competitive immunoassays or with polyclonal antibodies. In the present study, 24 monoclonal antibodies were raised against native or recombinant human CgA. Their mapping with proteolytic peptides showed that they defined eight distinct epitopic groups which spanned two-thirds of the C-terminal part of human CgA. All monoclonal antibodies were tested by pair and compared with a reference radioimmunoassay (RIA) involving CGS06, one of the monoclonal antibodies against the 198-245 sequence. It appears that $\mathrm{CgA}$ C-terminal end seems to be highly affected by proteolysis and the association of C-terminal and median-part monoclonal antibodies is inadequate for total $\mathrm{CgA}$ assessment. Our new immunoradiometric assay involves two monoclonal antibodies, whose contiguous epitopes lie within the median 145-245 sequence. This assay allows a sensitive detection of total human CgA and correlates well with RIA because dibasic cleavage sites present in the central domain do not seem to be affected by degradation. It has been proved to be efficient in measuring CgA levels in patients with neuroendocrine tumours.
\end{abstract}

Keywords: chromogranin A; monoclonal antibodies; epitope mapping; proteolysis; immunoradiometric assay

Human chromogranin $\mathrm{A}(\mathrm{hCgA})$ is a $48-\mathrm{kDa}$ protein with a $\mathrm{pI}$ of 4.9, encompassing 439 amino acids (Konecki et al, 1987). It belongs to the granin family with which it shares numerous structural and physiological similarities (Simon and Aunis, 1989 for a review). $\mathrm{CgA}$ is largely distributed in secretory granules of endocrine and neuroendocrine cells and constitutes, with other members of the granin family, one of their most abundant components (Cetin and Grube, 1991).

It has been shown that $\mathrm{CgA}$ plays an essential prohormone role through the release of bioactive peptides produced by its intragranular and extracellular proteolysis of the protein (Barbosa et al, 1991; Metz-Boutigue et al, 1993). Many studies have described natural peptides in human, as well as in bovine samples, including pancreastatin, vasostatins/ $\beta$-granin, chromostatin, WE-14, parastatin/GE-25 (Iacangelo and Eiden, 1995 for a review) and prochromacin (Strub et al, 1996). These peptides display several biological properties, including antibacterial activity as recently demonstrated by Strub et al (1996) for bovine prochromacin. The proteolysis concerns mainly the ten dibasic sites distributed along the $\mathrm{hCgA}$ sequence, although other types of cleavage have been described elsewhere on the sequence (Metz-Boutigue et al, 1993). It has also been shown that this process is recurrent for $\mathrm{C}$ - and $\mathrm{N}$ terminal ends (Barbosa et al, 1991; Metz-Boutigue et al, 1993).

Received 8 January 1998

Revised 3 April 1998

Accepted 7 April 1998

Correspondence to: F Degorce
Furthermore, several studies demonstrated that $\mathrm{CgA}$ could be degraded in a tissue-specific manner, which led to important disparities in the tissular distribution of the peptides released (Cetin and Grube, 1991; Curry et al, 1991; Watkinson et al, 1991). It is likely that the proteolysis properties may be responsible for the variability of the fragments found in normal and pathological tissues, blood or urine. Corti et al (1996a) recently confirmed this diversity by demonstrating the occurrence of different antigenic profiles among a population of patients affected with phaeochromocytoma. Apart from its differential expression in normal and neoplastic tissues, many studies have demonstrated the diagnostic value of $\mathrm{hCgA}$ measurement in blood. Levels of total circulating hCgA are significantly elevated in phaeochromocytomas, carcinoid and pancreatic endocrine tumours (O'Connor and Deftos, 1986). These data have been confirmed and extended to other neoplasia: neuroblastoma and gastrointestinal tumours (Eriksson et al, 1990; Hsiao et al, 1990; Stridsberg et al, 1995). Other authors have also described how the occurrence of plasma $\mathrm{hCgA}$ in prostatic carcinoma may be the sign of an unfavourable evolution (Cussenot et al, 1996; Deftos et al, 1996).

Circulating $\mathrm{CgA}$ is usually determined by competition assays, similar to the immunoassay described by O'Connor and Bernstein (1984), either with radiolabelled or with enzyme-conjugated CgA (Dillen et al, 1989). More recently, sandwich methods involving monoclonal or polyclonal antibodies have been published (Bender et al, 1992; Syversen et al, 1994; Corti et al, 1996a).

The proteolysis of $\mathrm{CgA}$ and the multiplicity of circulating fragments suppose an immunoassay configuration able to detect most, 
if not all, of these entities. The aim of the present study was to develop a sandwich assay which would enable the measurement of intact and fragmented circulating human $\mathrm{CgA}$. We assumed that a combination of monoclonal antibodies (mAbs) directed against the median part of the protein, presumably less exposed to proteolysis because of post-translational modifications (Strub et al, 1997), would satisfy such requirements. We therefore generated and selected 24 mAbs against hCgA (human native chromogranin A) and $\mathrm{rhCgA}$ (recombinant human chromogranin A). Epitope mapping using both Biacore and hCgA proteolytic peptides was carried out to specifically address the different epitopes to the $\mathrm{CgA}$ protein. Screening of the different $\mathrm{mAb}$ pairs against normal and pathological sera led us to select a sandwich assay, which did not appear to be affected by $\mathrm{hCgA}$ proteolysis because the two mAbs involved possess contiguous epitopes in the central part of $\mathrm{hCgA}$. Finally, this new assay has been proved to be efficient in measuring $\mathrm{CgA}$ levels in patients with neuroendocrine tumours.

\section{MATERIALS AND METHODS}

\section{Purification of CgAs}

Human adrenal phaeochromocytoma was used to purify hCgA according to the method described previously by O'Connor et al (1984). Final protein separation was carried out by 2D-gel electrophoresis according to O'Farrell's original technique (O'Farrell, 1975) modified by Bader and Aunis (1983). CgA was then electroeluted from gels (Metz-Boutigue et al, 1993). Escherichia coli BL21 (DE3) strain expressing rhCgA was grown as described elsewhere (Taupenot et al, 1995). Production of $\operatorname{rhCgA}$ was carried out according to the method described by the same authors.

\section{Radioiodination of purified $\mathrm{CgA}$}

Purified native or recombinant CgAs were radiolabelled with ${ }^{125} \mathrm{I}$ labelled sodium iodide according to the chloramine $\mathrm{T}$ method (Hunter and Greenwood, 1962) at a specific activity of $1500 \mathrm{kBq} \mu \mathrm{g}^{-1}$. Free iodine was removed by gel filtration on a Sephadex-G25 gel (Pharmacia Biotech, Orsay, France). Radiolabelled protein fractions were pooled and diluted in phosphatebuffered saline (PBS) pH 7.2 containing $45 \mathrm{mmol} \mathrm{l}^{-1}$ sodium azide and $0.5 \%$ bovine serum albumin (BSA).

\section{Immunizations}

Balb/c mice, 6-8 weeks old, were respectively immunized intraperitoneally with $10 \mu \mathrm{g}$ of purified $\mathrm{hCgA}$ or $\operatorname{rhCgA}$ in Freund's complete adjuvant. The following intraperitoneal doses were made in Freund's incomplete adjuvant at 1-month intervals. Immunizations of mice were monitored by serum titration via immunoprecipitation of $\left[{ }^{125} \mathrm{I}\right] \mathrm{hCgA}$. All animals were handled according to French law.

\section{Production and screening of hybridomas}

The mice were boosted intravenously with $10 \mu \mathrm{g}$ of hCgA in $0.9 \%$ sodium chloride solution 3 days before cell fusion. Spleen cells were isolated and fused with myeloma P3-X63 Ag8.653 cell line in the presence of $37 \%$ polyethylene glycol (MW 1540). Positive clones were identified by immunoprecipitation of $\left[{ }^{125} \mathrm{I}\right] \mathrm{hCgA}$. $\mathrm{mAbs}$ from positive clones were produced from mouse ascites and purified using protein A-Sepharose (Pharmacia Biotech).

\section{Mapping of monoclonal antibodies}

The different families of $\mathrm{CgA}$ epitopes were identified by testing the possible combinations of mAbs on the Biacore (Biacore, Saint Quentin-en-Yvelines, France) according to the manufacturer's instructions. Biacore is a system for realtime biomolecular interaction analysis based on surface plasmon resonance. Briefly, the sensorchips were prepared by coupling anti-mouse antibodies (CIS biointernational, Bagnols-sur-Cèze, France) with $N$-hydroxysuccinimide and water-soluble carbodiimide before sequential injection of the following reagents in $200 \mu \mathrm{l}$ of HEPES buffered saline $50 \mathrm{~mm}$, EDTA $15 \mathrm{~mm}$, sodium chloride $0.75 \mathrm{M}, \mathrm{pH}$ 7.4: (i) solid phase antibody, (ii) $\mathrm{rhCgA}$ at $20 \mu \mathrm{g} \mathrm{ml} \mathrm{m}^{-1}$, (iii) mixture of normal mouse immunoglobulins for saturation at $200 \mu \mathrm{g} \mathrm{ml}^{-1}$ and (iv) detecting antibody. All mAbs were injected at $50 \mu \mathrm{g} \mathrm{ml}^{-1}$. The sensorchip was regenerated with $0.1 \mathrm{mmol} \mathrm{l}^{-1}$ hydrochloric acid. Signals expressed in resonance units (RU) allowed us to work out epitope compatibilities and the performance of the different pairs.

\section{Mapping with rhCgA-derived peptides}

Recombinant hCgA ( $2 \mathrm{nmol})$ was digested for $2 \mathrm{~h}$ at $37^{\circ} \mathrm{C}$ with endoproteinase Lys-C or trypsin at a protein/proteinase ratio of 1000:1 in $100 \mathrm{mmol} \mathrm{l}^{-1}$ Tris- $\mathrm{HCl}, \mathrm{pH}$ 8.3. Generated rhCgAderived peptides were then separated on a Macherey Nagel 300$5 \mathrm{C} 18$ column $(250 \mathrm{~mm} \times 4 \mathrm{~mm})$. Absorbance was monitored at $214 \mathrm{~nm}$, and the solvent system consisted of $0.1 \%$ trifluoroacetic acid in water (solvent $\mathrm{A}$ ) and $0.1 \%$ trifluoroacetic acid in acetonitrile (solvent B). Material was eluted at a flow rate of $0.7 \mathrm{ml} \mathrm{min}-1$ using successive gradients of solvent B in A of $0-25 \%$ for $10 \mathrm{~min}$ and of $25-75 \%$ for $50 \mathrm{~min}$. Each peak fraction was collected and concentrated by evaporation, but not to complete dryness.

Aliquots of digested rhCgA high-performance liquid chromatography (HPLC) fractions were dotted on nitrocellulose sheets. Membranes were quickly washed with sodium chloride/inorganic phosphate (25 mM sodium phosphate $\mathrm{pH} 7.5$ containing $0.9 \%$ sodium chloride) and incubated for $2 \mathrm{~h}$ at room temperature with mAbs diluted $1: 1000$ in sodium chloride/ inorganic phosphate. The second antibody was an anti-mouse IgG conjugated to alkaline phosphatase (Biorad, Ivry-sur-Seine, France). Enzymatic reaction took place in $100 \mathrm{mmol} \mathrm{1}^{-1}$ Tris- $\mathrm{HCl}$ $\mathrm{pH} 8.5,100 \mathrm{mmol} \mathrm{l}^{-1}$ sodium chloride, $50 \mathrm{mmol} \mathrm{l}^{-1}$ magnesium chloride containing $0.4 \mathrm{mmol} \mathrm{l}^{-1}$ nitro-blue tetrazolium and $0.38 \mathrm{mmol} \mathrm{l}^{-1}$ 5-bromo-4-chloro-3-indolyl phosphate (Boehringer Mannheim, Meylan, France).

The sequence of purified peptides was determined by automatic Edman degradation on an Applied Biosystems 473A microsequencer. Samples purified by HPLC were loaded on polybrenetreated and precycled glass-fibre filters (Metz-Boutigue et al, 1993). Phenylthiohydantoin amino acids were identified by chromatography on a C-18 column (PTH C-18, $2.1 \mathrm{~mm} \times 200 \mathrm{~mm}$ ). Mass spectra analysis (Maldi-Tof) was obtained according to the procedure previously reported (Goumon et al, 1996).

\section{Reference radioimmunoassay for $\mathrm{hCgA}$}

mAb CGS06 against hCgA was used to design this RIA (radioimmunoassay). Purified rhCgA used for the calibrators was diluted in normal human serum. The calibrator concentrations ranged from 0 up to $1600 \mathrm{ng} \mathrm{ml}^{-1}$. After combining $50 \mu \mathrm{l}$ of calibrator or sample to be assayed, $300 \mu \mathrm{l}$ of radiolabelled $\mathrm{hCgA}$ 
Table 1 Characteristics of mAbs obtained. Names in bold type indicate antibodies raised against native hCgA. Epitope mapping as well as stoichiometry which is expressed as mole of $\mathrm{rhCgA} / \mathrm{mole}$ of $\mathrm{mAb}$ were carried out with Biacore. mAb CGSO3 stoichiometry could not be tested (n.t.)

\begin{tabular}{|c|c|c|c|}
\hline Name & Isotype & Stoichiometry & Epitope group \\
\hline CGS01 & $\operatorname{lgG} 1$ & 0.431 & 1 \\
\hline CGS03 & $\lg G 1$ & n.t. & \\
\hline CGS04 & $\lg G 2 b$ & 0.365 & \\
\hline CGS12 & $\operatorname{lgG} 1$ & 0.364 & \\
\hline CGS06 & $\lg G 2 a$ & 0.503 & 2 \\
\hline CGS08 & $\lg G 1$ & 0.457 & \\
\hline CGS10 & $\operatorname{lgG} 1$ & 0.182 & $3 a$ \\
\hline CGS05 & $\lg \mathrm{I} 1$ & 0.157 & \\
\hline CGS23 & $\lg G 2 a$ & 0.604 & \\
\hline CGS24 & $\lg G 2 a$ & 0.521 & \\
\hline CGS25 & $\lg G 2 a$ & 0.553 & \\
\hline CGS26 & $\lg G 2 a$ & 0.356 & \\
\hline CGS29 & $\lg G 1$ & 0.621 & $3 b$ \\
\hline CGS34 & $\lg G 1$ & 0.536 & \\
\hline CGS35 & $\lg G 1$ & 0.539 & $3 c$ \\
\hline CGS30 & $\lg G 2 a$ & 0.327 & 4 \\
\hline CGS31 & $\lg \mathrm{I} 1$ & 0.198 & \\
\hline CGS33 & $\lg G 1$ & 0.383 & \\
\hline CGS36 & $\lg G 1$ & 0.184 & \\
\hline CGS17 & $\lg \mathrm{G} 1$ & 0.162 & 5 \\
\hline CGS19 & $\lg G 1$ & 0.361 & \\
\hline CGS20 & $\lg G 1$ & 0.254 & 6 \\
\hline CGS21 & $\lg G 1$ & 0.183 & 7 \\
\hline CGS32 & $\lg \mathrm{I} 1$ & 0.262 & 8 \\
\hline
\end{tabular}

( $2 \mathrm{kBq}$ per tube) and $150 \mu \mathrm{l}$ of purified CGS06 mAb solution ( 80 $\mathrm{ng} \mathrm{ml}{ }^{-1}$ ) in a tube, the first incubation took place for $24 \mathrm{~h}$ at room temperature under gentle shaking. Bound complexes were separated for $20 \mathrm{~min}$ at room temperature by adding $75 \mu 1$ of sheep anti-mouse immunoglobulins diluted to $1: 10$ in PBS and $50 \mu 1$ of human normal serum. One millilitre of $6 \%$ polyethylene glycol (MW 6000) was added and pellets were counted on a $\gamma$-counter (Crystal II, Packard Instrument, Rungis, France) after centrifuging at $2000 \mathrm{~g}$ for $15 \mathrm{~min}$ (961R, Seroa, Monaco).

\section{Selection of a suitable pair of mAbs for immunoradiometric assays (IRMA)}

mAbs whose epitopes had been mapped to hCgA were used for the screening of an optimal antibody combination for the measurement of CgA. All pairs were tested against two pathological plasma pools from several patient samples with phaeochromocytoma (PP) and carcinoids (CP) previously assayed in the RIA, as well as against a pool of plasmas coming from healthy individuals (NP).

Three combinations that involved epitopes spanning the Cterminal end of the protein (CGS29 and CGS04) were retained for further testing on a larger population of normal subjects $(n=20)$ and of 39 patients suffering from neuroendocrine tumours: phaeochromocytoma, bronchial and intestinal carcinoids, pancreatic endocrine neoplasia, medullary thyroid carcinoma (MTC). All plasmas were obtained and used in accordance with local ethical rules.

\section{Experimental procedure}

All antibodies were brought to a concentration of $10 \mu \mathrm{g} \mathrm{ml}^{-1}$ in PBS pH 7.5 and coated to polystyrene tubes (Greiner France, Poitiers, France). Concurrently, mAbs were radiolabelled with ${ }^{125}$ I-labelled sodium iodide using the chloramine $\mathrm{T}$ method as

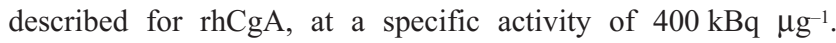
Calibrators were made as described earlier for RIA. Assay buffer consisted of disodium hydrogen phosphate/potassium dihydrogen phosphate $50 \mathrm{mmol} \mathrm{l}^{-1}$, EDTA $1 \mathrm{mmol} \mathrm{l}^{-1}$, sodium azide $15 \mathrm{mmol} \mathrm{1}^{-1}$, BSA 1\%, pH 7.0. Briefly, $1 \mathrm{ml}$ of assay buffer and $50 \mu 1$ of standard or sample were mixed in coated tubes. Incubation took place for $18 \mathrm{~h}$ at room temperature. Tubes were washed twice with $2 \mathrm{ml}$ of a solution of $0.3 \%$ Tween 20 . One millilitre of radiolabelled $\mathrm{mAb}$ was then added to each tube and incubated for $2 \mathrm{~h}$ at room temperature under gentle agitation. Tubes were washed again as previously described and radioactivity was counted on the $\gamma$-counter.

\section{RESULTS}

\section{Purification of $\mathrm{CgA}$}

After microsequencing, the degree of purity of $\mathrm{CgA}$ preparations was checked by 2D-gel electrophoresis as described by MetzBoutigue et al (1993). The two-dimensional gel showed a major spot (70 kDa; pI 5-5.5) corresponding to intact $\mathrm{CgA}$ and minor $\mathrm{CgA}$-derived fragments, the latter representing $5 \%$ of total protein. This pattern of the gel was consistent with results obtained by Taupenot et al (1995).

\section{Generation of monoclonal antibodies}

Two generations of antibodies were obtained with $\mathrm{hCgA}$ and rhCgA respectively (Table 1). Eleven cell fusions were carried out with mice immunized with hCgA, producing eight hybridomas. The two cell fusions with animals immunized against the recombinant protein generated 16 different clones. A total of 17 IgG1, six $\operatorname{IgG} 2 \mathrm{a}$ and one $\operatorname{IgG} 2 \mathrm{~b}$ were produced for further characterization.

\section{Biacore mapping}

All possible pairs of $\mathrm{mAbs}$ were tested with Biacore using rhCgA. The $24 \mathrm{mAbs}$ could be distributed into eight distinct groups (Table 1). mAbs from group 3, however, could be further subdivided because of partial inhibitions existing between CGS10-like antibodies and CGS29, CGS34 and CGS35.

Stoichiometry was determined for each $\mathrm{mAb}$ by calculation of the ratio RU (resonance units) of bound $\mathrm{rhCgA} / \mathrm{RU}$ of immobilized $\mathrm{mAb}$. Values were found to range from 0.157 to $0.621 \mathrm{~mole}$ $\mathrm{rhCgA} /$ mole $\mathrm{mAb}$. No obvious relationship could be established between the type of immunogen used and the stoichiometry, except for mAbs from group 3a. In this group, the stoichiometry of CGS10 and CGS05 raised against human native CgA is significantly weaker than that of second generation mAbs against the recombinant protein.

One $\mathrm{mAb}$ of each main group was retained for further characterization: CGS04, CGS06, CGS10, CGS17, CGS20, CGS21, CGS30 and CGS32 were selected. In spite of a weaker stoichiometry, CGS10 and CGS17 were also included in this series because of better hybridoma productivity. 
A

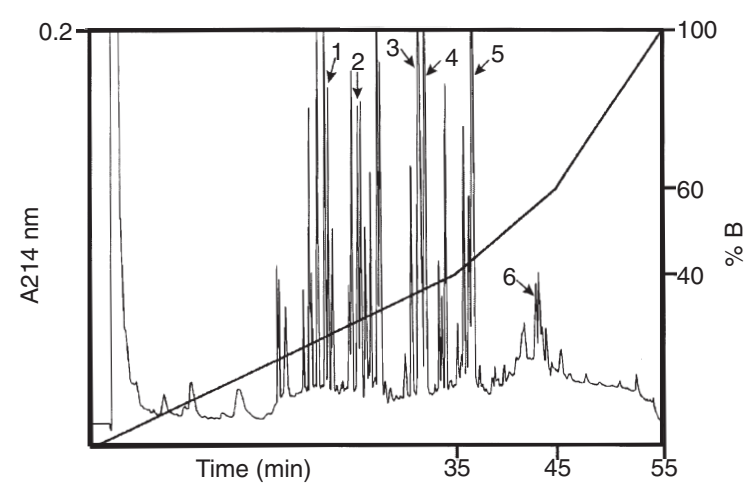

B

\begin{tabular}{cclcc}
\hline Fraction & Antibody & N-terminal sequence & Mass (Da) & Fragment \\
\hline 1 & CGS17 & RLEGQEE & 2053.9 & $339-355$ \\
2 & CGS30 & AEGNNQA & 5635.8 & $145-197$ \\
3 & CGS20 & EIRKGESRS & 6224.8 & $246-303$ \\
4 & CGS06 & GLSAEPG & 5265.4 & $198-245$ \\
5 & CGS21 & LXFRARA & 5145.8 & $356-400$ \\
6 & CGS32 & GXTEVMKXIV & 43532 & $10-400$ \\
$*$ & CGS04 & LEGQEE & n.d. & $340-394$ \\
$*$ & CGS10 & GYPEEK & n.d. & $395-439$ \\
\hline
\end{tabular}

Figure 1 (A) HPLC profile of digested rhCgA with endoprotease Lys-C. (B) Characteristics of fragments recognized by the different mAbs. mAbs CGSO4 and CGS10 were tested by Western blot and the corresponding immunodetected spot was sequenced according to Metz-Boutigue et al (1993). n.d., mass spectroscopy was not carried out for these mAbs; X, non-identified residue. Location of $\mathrm{CgA}$-derived fragments was obtained according to $\mathrm{CgA}$ sequence (Koneki et al, 1987)

\section{Mapping of monoclonal antibodies after endoproteinase Lys-C digestion of rhCgA}

Recombinant hCgA was digested with endoproteinase Lys-C and all the mAbs mentioned above were tested against each HPLC aliquot fraction of digested rhCgA (Figure 1). mAbs CGS17 and CGS21 recognized the C-terminal domain of the protein with a high specificity for regions 339-355 and 356-400 respectively (Figure 2). In addition, mAbs CGS04 and CGS10 were directly tested by Western blot, and the immunodetected fragments were in the C-terminal region of CgA (340-394 and 395-439). mAbs CGS06, CGS20 and CGS30 were specific for the median part of the protein core 198-245, 246-303 and 145-197 (Figure 2). Finally, mAb CGS32 was strongly reactive with a large fragment 10-400; this antibody immunodetected a central fragment corresponding to sequence $198-245$, but reaction was weak.

\section{Selection of a suitable mAb pair}

The two pathological pools (PP and CP) were made from several EDTA plasmas coming respectively from patients with phaeochromocytoma and intestinal carcinoids. CgA levels, as determined by the reference RIA, were found to be $675 \mathrm{ng} \mathrm{ml}^{-1}$ for PP and $1600 \mathrm{ng} \mathrm{ml}^{-1}$ for CP. These values were compared with that of the normal pool which was determined to be $40 \mathrm{ng} \mathrm{ml}^{-1}$ by the reference RIA.

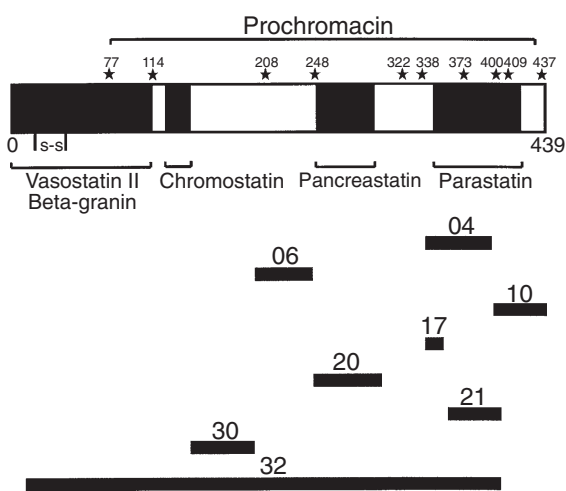

Figure 2 Human $\mathrm{CgA}$ sequence and position of the different epitopes. Stars and corresponding numbers indicate the dibasic cleavage sites. mAb CGS32 epitope could not be identified precisely (10-400)

The results for the $56 \mathrm{mAb}$ combinations tested (Table 2) show the values obtained for the highest rhCgA calibrator at $1200 \mathrm{ng} \mathrm{ml}^{-1}$, as well as the concentration ratios for PP/NP and $\mathrm{CP} / \mathrm{NP}$. For 26 combinations, $\mathrm{CP}$ was out of range and had to be further diluted. This was also the case for PP in three combinations.

Combinations using CGS21 as a tracer hardly detect rhCgA, as shown by a bound/total ratio of less than $5 \%$ at the $1200 \mathrm{ng} \mathrm{ml}^{-1}$ calibrator. This was also the case, to a minor extent, for tracer CGS10 (three combinations out of seven and low B/T in general). All other systems were able to detect rhCgA and showed a significant rise in the signal with increasing rhCgA concentrations.

Concerning the detection of $\mathrm{CgA}$ in pathological pools, several types of combinations emerge. First, several systems gave a negative or a poor discrimination of the pools, i.e. CgA level measured in PP was never greater than twice that of NP. This situation applied to most of the combinations involving CGS10 and CGS04, either immobilized or used as tracers. Systems involving CGS17 or CGS20 gave a weaker discrimination, especially when associated with antibodies against the 340-439 region of the protein (CGS17, CGS21, CGS04 and CGS10). This was particularly true when the two mAbs were used as capture antibodies.

A second behaviour could be identified through systems such as CGS21/CGS06*, CGS21/CGS30* or CGS32/CGS06* which discriminated the two pathological pools positively but did not differentiate them from each other.

Finally, 19 combinations showed PP/NP ratios of greater than 2 as well as high $\mathrm{CP} / \mathrm{NP}$ ratios, with substantially elevated $\mathrm{CgA}$ levels in CP italic data in Table 2). When the combination of CGS21/CGS32* is excluded (i.e., the system could not be reversed), it seems that only heterologous combinations involving mAbs CGS06, CGS30 and CGS32 were able to measure increasing $\mathrm{CgA}$ concentrations in NP, PP and $\mathrm{CP}$ with results close to the reference RIA. All combinations selected involved epitopes mapped to the median part of the protein.

To further check these conclusions, three different systems using tracer mAbs spanning the C-terminal domain of the protein were tested on a larger population of samples (Figure 3) and compared with the reference RIA. Because mAb CGS10 behaved poorly as a tracer when associated with CGS06, mAb CGS29, which shares the same epitope as CGS10, was used for this experiment.

Analysis of the pathological/normal ratios at the 95th percentile by Wilcoxon matched-pairs signed-ranks showed that 
Table 2 Evaluation of the different mAb combinations on normal vs pathological plasma pools

\begin{tabular}{|c|c|c|c|c|c|c|c|c|}
\hline \multirow{2}{*}{$\begin{array}{l}\text { Solid } \\
\text { phases }\end{array}$} & \multicolumn{8}{|c|}{ Tracers } \\
\hline & CGS32 & CGS30 & CGS06 & CGS20 & CGS17 & CGS21 & CGSO4 & CGS10 \\
\hline CGS32 & & $\frac{\frac{48.13^{a}}{16.4^{b}}}{\frac{23.3^{c}}{\underline{3}^{2}}}$ & $\begin{array}{c}30.89 \\
24.3 \\
28.1\end{array}$ & $\frac{7.01}{\frac{8.8}{50.3}}$ & $\frac{15.63}{\underline{4.3}}$ & $\begin{array}{l}3.01 \\
18.0 \\
39.6\end{array}$ & $\begin{array}{c}32.17 \\
1.9 \\
15.6\end{array}$ & $\begin{array}{c}3.53 \\
1.0 \\
9.3\end{array}$ \\
\hline CGS30 & $\frac{21.56}{\frac{10.9}{17.7}}$ & & $\frac{57.56}{\underline{16.0}}$ & $\frac{22.04}{\frac{5.6}{21.8}}$ & $\frac{\underline{29.2}}{\underline{3.0}}$ & $\begin{array}{l}3.38 \\
14.4 \\
31.3\end{array}$ & $\begin{array}{c}\mathbf{6 0 . 5 7} \\
1.2 \\
9.3\end{array}$ & $\begin{array}{c}9.40 \\
0.5 \\
7.5\end{array}$ \\
\hline CGS06 & $\frac{\frac{18.8}{11.3}}{18.5}$ & $\begin{array}{l}\frac{65.51}{\underline{9.9}} \\
\underline{18.2}\end{array}$ & & $\begin{array}{l}\frac{12.8}{4.0} \\
\underline{23.2}\end{array}$ & $\begin{array}{c}20.38 \\
1.6 \\
22.0\end{array}$ & $\begin{array}{c}2.6 \\
8.0 \\
18.5\end{array}$ & $\begin{array}{c}\mathbf{4 5 . 1 7} \\
1.0 \\
10.6\end{array}$ & $\begin{array}{c}3.92 \\
0.9 \\
3.8\end{array}$ \\
\hline CGS20 & $\begin{array}{c}15.11 \\
1.3 \\
10.2\end{array}$ & $\begin{array}{l}\frac{43.22}{2.6} \\
\underline{12.8}\end{array}$ & $\begin{array}{l}\frac{25.43}{2.0} \\
\underline{15.6}\end{array}$ & & $\begin{array}{c}8.29 \\
1.7 \\
26.6\end{array}$ & $\begin{array}{c}1.42 \\
2.5 \\
11.3\end{array}$ & $\begin{array}{c}30.70 \\
0.8 \\
7.6\end{array}$ & $\begin{array}{c}1.61 \\
0.0 \\
0.9\end{array}$ \\
\hline CGS17 & $\begin{array}{l}\frac{17.67}{2.3} \\
\underline{14.2}\end{array}$ & $\begin{array}{l}\frac{62.66}{\underline{2.4}} \\
\underline{22.4}\end{array}$ & $\begin{array}{l}\frac{49.52}{\underline{2.3}} \\
\underline{24.0}\end{array}$ & $\begin{array}{l}\underline{21.29} \\
\underline{2.0} \\
\underline{20.9}\end{array}$ & & $\begin{array}{c}2.7 \\
2.4 \\
20.7\end{array}$ & $\begin{array}{c}\mathbf{6 0 . 8 6} \\
1.0 \\
10.4\end{array}$ & $\begin{array}{c}7.81 \\
0.5 \\
5.4\end{array}$ \\
\hline CGS21 & $\frac{15.86}{\frac{9.6}{20.4}}$ & $\begin{array}{c}\mathbf{6 7 . 4 6} \\
13.0 \\
14.6\end{array}$ & $\begin{array}{c}\mathbf{5 0 . 5 2} \\
16.2 \\
16.2\end{array}$ & $\frac{\frac{15.4}{5.5}}{\frac{11.7}{1.7}}$ & $\begin{array}{c}\frac{27.53}{3.3} \\
\underline{19.4}\end{array}$ & & $\begin{array}{c}\mathbf{4 8 . 5 7} \\
1.1 \\
5.5\end{array}$ & $\begin{array}{c}7.82 \\
0.0 \\
0.7\end{array}$ \\
\hline CGSO4 & $\begin{array}{c}28.83 \\
1.1 \\
4.7\end{array}$ & $\begin{array}{c}76.53 \\
1.2 \\
13.5\end{array}$ & $\begin{array}{c}55.53 \\
1.1 \\
28.2\end{array}$ & $\begin{array}{c}\mathbf{2 6 . 7 1} \\
1.1 \\
24.3\end{array}$ & $\begin{array}{c}\mathbf{5 5 . 0 5} \\
0.9 \\
17.3\end{array}$ & $\begin{array}{c}3.62 \\
1.2 \\
14.3\end{array}$ & & $\begin{array}{c}19.57 \\
0.7 \\
16.4\end{array}$ \\
\hline CGS10 & $\begin{array}{c}12.39 \\
0.1 \\
3.9\end{array}$ & $\begin{array}{c}33.96 \\
0.7 \\
5.0\end{array}$ & $\begin{array}{c}17.49 \\
0.8 \\
9.0\end{array}$ & $\begin{array}{c}7.77 \\
0.5 \\
6.1\end{array}$ & $\begin{array}{c}11.65 \\
0.5 \\
4.0\end{array}$ & $\begin{array}{c}1.44 \\
0.9 \\
4.0\end{array}$ & $\begin{array}{c}30.54 \\
0.6 \\
4.3\end{array}$ & \\
\hline
\end{tabular}

aPer cent binding at $1200 \mathrm{ng} \mathrm{ml}^{-1}$ of rhCgA. ${ }^{\mathrm{b}}$ and ${ }^{\mathrm{C}} \mathrm{CgA}$ concentration ratios PP/NP and CP/NP respectively. Dark grey squares indicate low-sensitivity systems. Light grey squares stand for negative or poor discriminative systems. White squares with underlined italic type show combinations that discriminate PP and CP positively as well as one from the other. Ratios of PP/NP and CP/NP were found to be 16.9 and 40 in the reference RIA.
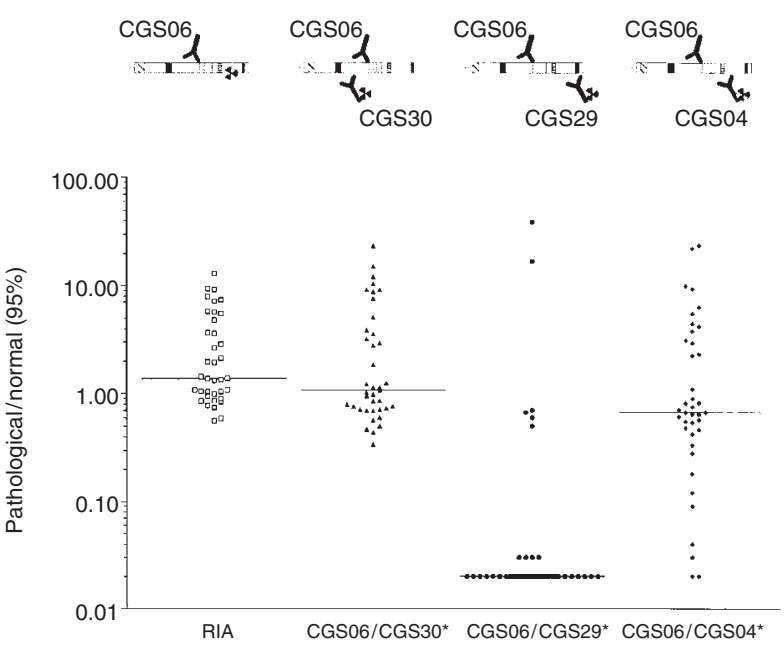

Figure 3 Comparison of three different immunoradiometric assays vs the reference RIA. Data are expressed as the concentration ratio of pathologica plasma/95th percentile value of normal population $(n=20)$. Line segments indicate the median value for each group

CGS06/CGS30* and the reference RIA, as well as CGS06/CGS04* and the RIA, are closely related $(P<0.0001$ and $P=0.011$ respectively). In contrast, CGS06/CGS29* is significantly different from the RIA $(P=0.738)$. The CGS06/CGS30* combination with two contiguous epitopes within the median part of CgA gave a very similar distribution of the samples when compared with the RIA. Normal values ranged from 33 to $94 \mathrm{ng} \mathrm{ml}^{-1}$ and the cut-off value was fixed at $86 \mathrm{ng} \mathrm{ml}^{-1}$ (95th percentile of normal population). However, the use of CGS29 tracer, whose epitope is CGS10-like, led to a dramatic decrease in the concentrations measured. The rhCgA calibrator at $1200 \mathrm{ng}$ $\mathrm{ml}^{-1}$ was detected at a significant level $(20 \%$ of B/T). However, concentrations measured for normal plasmas ranged from 0 to $55 \mathrm{ng} \mathrm{ml}^{-1}$ and $\mathrm{CgA}$ level was undetectable in $50 \%$ of the population. With this system, only two samples could be measured above the cut-off $\left(30 \mathrm{ng} \mathrm{ml}^{-1}\right)$ and they both came from patients with intestinal carcinoids. Furthermore, 29 samples out of 39 had an undetectable level of $\mathrm{CgA}$, irrespective of their origin.

When an intermediate mAb such as CGS04 was used, the dispersion was improved although the concentrations of 8 samples out of 39 were clearly diminished, including two samples with no CgA. All samples from healthy individuals could be detected (range 15-71 $\mathrm{ng} \mathrm{ml}^{-1}$ ) and enabled us to fix the cut-off value at 67 ng ml ${ }^{-1}$. All these results clearly show that CGS06/CGS30* is the closest combination to the reference RIA.

\section{DISCUSSION}

In the present study, 24 monoclonal antibodies were generated against native and recombinant human $\mathrm{CgA}$, and we report here their characterization and the development of a sandwich IRMA for hCgA. 
The epitope analysis carried out raises several questions. The experiment for the selection of a mAb pair showed that CGS04 perfectly detected rhCgA contained in calibrators, but failed to recognize $\mathrm{hCgA}$ in $\mathrm{PP}$ when associated to any other $\mathrm{mAb}$ and irrespective of its position in the system. Similarly to CGS10, which shows the same behaviour, it appears, therefore, that the proteolysis recurrently affecting both ends of the protein (Gill et al, 1992; Metz-Boutigue et al, 1993) is particularly active against the last three, perhaps four, cleavage sites $(373,400,409$ and 437). It is important to stress that the processing is complex and governed by many different aspects. Among those, Strub et al (1997) showed that proteolysis of bovine $\mathrm{CgA}$ could depend on post-translational modifications. For instance, the putative cleavage site 373-374 may or may not constitute a proteolytic target, depending on the phosphorylation of serine-380. In contrast, CGS21, whose epitope considerably overlaps that of CGS04, behaves quite differently, and its association with antibodies against the median part of hCgA enables the detection of PP. Given the length of the sequences deduced, it is most likely that the actual CGS04 epitope is located in the C-terminal end of the 340-394 region; while CGS21 recognizes the N-terminal part of the 356-400 sequence, the cleavage site 373-374 is lying in between. The nature of the CGS17 epitope remains unclear. Mapping with rhCgA peptides indicates a very restricted sequence (339-355), upstream of the CGS21 epitope. Surprisingly, CGS17 barely detects PP when associated with median-type antibodies. Cleavage sites located upstream do not seem to be involved either, as shown by a positive association of CGS21 with the same antibodies. Strub et al (1997) have described a phosphorylation site on serine-307 of bovine $\mathrm{CgA}$ that is conserved in $\mathrm{hCgA}$ (ser-315) and, thus, close to the CGS17 epitope. Differential phosphorylation of $\mathrm{CgA}$ in normal or pathological tissues - through the activation of this mechanism in tumours - may explain such a difference in terms of immunoreactivity because the rhCgA against which CGS17 was raised is not post-translationally modified.

Median-type mAb epitopes (CGS20, CGS06, CGS30) are likely to recognize the $\mathrm{CgA}$ core as well. The three mAbs detect native and recombinant $\mathrm{hCgA}$ equally well, although CGS20 and, above all, CGS06 are close to two putative $O$-glycosylation targets (Strub et al, 1997). Finally, CGS32 could not be addressed accurately. Given its pattern (i.e. discrimination of the different pools), it is probably directed against the median part of CgA. All these results contradict to a certain extent the antigenic profiles already described by Gill et al (1992) and Corti et al (1996b). It first appears that most of the mAbs we obtained are directed against regions of $\mathrm{hCgA}$ which were shown to be poorly antigenic by these authors, apart from the CGS04 and CGS06 epitope groups that match respectively the highly antigenic domains 375-394 and 222-230. Unexpectedly, none of the mAbs detected the Nterminal third of the protein. This result remains unexplained because both immunogens used contained hardly any truncated $\mathrm{hCgA}$. However, this part of the protein is well preserved among different species and it may be less immunogenic.

As first published by O'Connor and Bernstein (1984), many $\mathrm{CgA}$ immunoassays involved radiolabelled $\mathrm{CgA}$ and polyclonal antibodies. In these configurations, $\mathrm{CgA}$ measurements reflect levels of intact as well as truncated $\mathrm{CgA}$. To detect most of these entities, CGS06, against the 198-245 sequence of hCgA, was chosen to develop a reference RIA. Results obtained with this assay on a population of various neuroendocrine neoplasia were consistent with previously described competition assays (data not shown). Following the same assumption, we wanted to check whether the association of CGS06 with other mAbs against $\mathrm{CgA}$ could allow the assessment of total $\mathrm{hCgA}$ in a comparable manner. The evaluation of all possible $\mathrm{mAb}$ combinations with three plasma pools showed that only those systems involving mAbs against the median domain of hCgA effectively discriminate. We excluded all the combinations that detected rhCgA weakly because the concentrations calculated may be inaccurate. For instance, all combinations with the tracer CGS21 were eliminated. In these cases, minor contaminations of calibrators by smaller rhCgA fragments could be suspected, although they cannot have such an influence in the second step of the assay. Moreover, combinations with CGS04 as tracer, whose epitope is immediately downstream from that of CGS21, perfectly detect increasing concentrations of $\mathrm{rhCgA}$. It is therefore very likely that radioiodination by chloramine $\mathrm{T}$ simply alters CGS21 immunoreactivity. CGS10 reactivity seems to be affected to a lesser extent (apart from in the CGS04/CGS10* system) for the same reasons. It must be stressed that this problem could be ruled out when CGS10 was replaced by CGS29, a mAb from the same group, for the last comparative test, which confirmed yet again the full integrity of the rhCgA calibrator.

Concerning the results obtained from the pathological pools, it is clear that a major degradation occurs at the C-terminal end of hCgA. Only combinations involving median-type mAbs against the large domain 145-303 allow a reliable discrimination especially when corresponding epitopes are contiguous, as for CGS06 and CGS30. Besides, these data confirm that the cleavage site 208 separating the two epitopes is relatively unaffected. The final test with systems associating immobilized CGS06 with other mAbs spanning the $\mathrm{CgA} \mathrm{C}$-terminal domain confirms yet again the assumptions made about the proteolysis of this region. The involvement of the last cleavage sites (400, 409 and 437) is obvious, not only with regard to the concentration measured in pathological plasmas but also in normals for which half the population assessed did not contain any hCgA. Only two samples from carcinoid tumours were highly positive and comparable to the levels obtained with the three other assays. However, this comparison could not allow us to conclude the exact extent to which the proteolysis occurs on the C-terminal domain, nor to establish a relationship with the type of pathology. The degradation is substantially less active upstream in the sequence, as shown by the widespread dispersion obtained with CGS06/CGS04*. There were, nevertheless, a fair number of samples for which the majority of circulating hCgA was apparently truncated from the 373-439 domain, and perhaps from a wider part. The extension of this study to the CGS06/CGS21* or CGS20* systems would be necessary to confirm which of the three cleavage sites $(322,338$ or 373) are processed preferentially.

The clinical value of $\mathrm{CgA}$ measurement relies on the detection of all its circulating forms. Although it could be speculated that the detection of a more restricted fragment could correlate to total CgA measurement, it has been shown that the assessment of plasma pancreastatin, produced by cleavage at the 243 and 294 dibasic sites, was less discriminative and of less clinical interest than that of total CgA (Stridsberg et al, 1995). Our results show that the hCgA C-terminal end is particularly affected by degradation, but that proteolysis decreases when moving upstream in the sequence. They confirm those of Metz-Boutigue et al (1993) who also found that this region is predominantly processed. Under these circumstances, a mAb against the less affected median part 
of hCgA (CGS06) behaves in a comparable manner to a polyclonal antibody, and thus allows the capture of a large number of $\mathrm{CgA}$ entities. The association of this $\mathrm{mAb}$ with another one having a contiguous epitope (CGS30) also enables the measurement of major fragments and intact hCgA. After these results, this immunoassay is readily included in several experimental protocols, in order to evaluate its clinical value on larger populations as well as on other neuroendocrine pathologies or mixed neoplasia. In a first comparative study, Baudin et al (1998) show that $\mathrm{CgA}$ was more sensitive than NSE in a population of patients with gastroenteropancreatic neuroendocrine tumours, MTC and phaeochromocytoma. The results obtained with this immunoradiometric assay also suggest that $\mathrm{CgA}$ may be more satisfactory than neuron-specific enolase for the follow-up of such patients.

\section{ACKNOWLEDGEMENTS}

We express our sincere gratitude to P Garcia-Sablone for help in the mapping of monoclonal antibodies and to M Moniatte for mass spectra analysis. We are indebted to Professors E Comoy (Institut Gustave Roussy, Villejuif, France) and D Guilloteau (Hôpital Bretonneau, Tours, France) for kindly providing the pathological plasmas. We are very grateful to B Marshall for reviewing this manuscript and to MH Garnier for her technical assistance.

\section{REFERENCES}

Bader MF and Aunis D (1983) The 97-Kd $\alpha$-actinin-like protein in chromaffin granule membranes from adrenal medulla: evidence for localization on the cytoplasmic surface and for binding to actin filaments. Neuroscience $\mathbf{8}$ : $165-181$

Barbosa JA, Gill BM, Takiyyuddin MA and O'Connor DT (1991) Chromogranin A: post-translational modifications in secretory granules. Endocrinology 128: 174-190

Baudin E, Gigliotti A, Ducreux M, Ropers J, Comoy E, Sabourin JC, Bidart JM, Cailleux AF, Bonacci R, Ruffié P and Schlumberger M (1998) Neuron-specific enolase and chromogranin A as markers of neuroendocrine tumors (in press)

Bender H, Maier A, Wiedenmann B, O'Connor DT, Messner K and Schmidt-Gayk H (1992) Immunoluminometric assay of chromogranin A in serum with commercially available reagents. Clin Chem 38: 2267-2272

Cetin Y and Grube D (1991) Topology of chromogranins in secretory granules of endocrine cells. Histochemistry 96: 301-310

Corti A, Gasparri A, Chen FX, Pelagi M, Brandazza A, Sidoli A and Siccardi AG (1996a) Characterisation of circulating chromogranin A in human cancer patients. Br J Cancer 73: 924-932

Corti A, Longhi R, Gasparri A, Chen FX, Pelagi M and Siccardi AG (1996b) Antigenic regions of human chromogranin A and their topographic relationships with structural/functional domains. Eur J Biochem 235: 275-280

Curry WJ, Johnston CF, Hutton JC, Arden SD, Rutherford NG, Shaw C and Buchanan KD (1991) The tissue distribution of rat chromogranin A-derived peptides: evidence for differential tissue processing from sequence specific antisera. Histochemistry 96: 531-538

Cussenot O, Villette JM, Valeri A, Cariou G, Desgrandchamps F, Cortesse A, Meria P, Teillac P, Fiet J and Leduc A (1996) Plasma neuroendocrine markers in patients with benign prostatic hyperplasia and prostatic carcinoma. J Urol 155 : $1340-1343$

Deftos LJ, Nakada S, Burton DW, Di Sant'Agnese PA, Cockett TK and Abrahamsson PA (1996) Immunoassay and immunohistology studies of chromogranin A as a neuroendocrine marker in patients with carcinoma of the prostate. Urology 48: 58-62

Dillen L, De Block J, Van Lear L and De Potter W (1989) Enzyme-linked immunosorbent assay for chromogranin A. Clin Chem 35: 1934-1938

Eriksson B, Arnberg H, Oberg K, Hellman U, Lundqvist G, Wernstedt C and Wilander E (1990) A polyclonal antiserum against chromogranin A and B - a new sensitive marker for neuroendocrine tumours. Acta Endocrinol 122: $145-155$

Gill BM, Barbosa JA, Hogue-Angueletti R, Varki N and O'Connor DT (1992) Chromogranin A epitopes: clues from synthetic peptides and peptide mapping. Neuropeptides 21: 105-118

Goumon Y, Strub JM, Moniatte M, Nullans G, Poteur L, Hubert P, Van Dorsselaer A, Aunis D and Metz-Boutigue MH (1996) The C-terminal biphosphorylated proenkephalin-A-(209-237)-peptide from adrenal medullary chromaffin granules possesses antibacterial activity. Eur J Biochem 235: 516-525

Hsiao RJ, Seeger RC, Yu AL and O'Connor DT (1990) Chromogranin A in children with neuroblastoma. J Clin Invest 85: 1555-1559

Hunter WM and Greenwood FC (1962) Preparation of iodine-131 labeled human growth hormone of high specific activity. Nature 194: 495-496

Iacangelo AL and Eiden LE (1995) Chromogranin A: current status as a precursor for bioactive peptides and a granulogenic/sorting factor in the regulated secretory pathway. Regul Pept 58: 65-88

Konecki DS, Benedum UM, Gerdes HH and Huttner WB (1987) The primary structure of human chromogranin A and pancreastatin. J Biol Chem 262: 17026-17030

Metz-Boutigue MH, Garcia-Sablone P, Hogue-Angeletti R and Aunis D (1993) Intracellular and extracellular processing of chromogranin-A. Determination of cleavage sites. Eur J Biochem 217: 247-257

O'Connor DT and Bernstein KN (1984) Radioimmunoassay of chromogranin A in plasma as a measure of exocytotic sympathoadrenal activity in normal subjects and patients with pheochromocytoma. New Engl J Med 311: 764-770

O'Connor DT and Deftos LJ (1986) Secretion of chromogranin A by peptideproducing endocrine neoplasms. $N$ Engl J Med 314: 1145-1151

O'Connor DT, Frigon RP and Sokoloff RL (1984) Human chromogranin A. Purification and characterization from catecholamine storage vesicles of human pheochromocytoma. Hypertension 6: 2-12

O'Farrell PH (1975) High resolution two-dimensional electrophoresis. J Biol Chem 72: $248-254$

Simon JP and Aunis D (1989) Biochemistry of the chromogranin A protein family. Biochem J 262: 1-13

Stridsberg M, Oberg K, Li Q, Engstrom U and Lundqvist G (1995) Measurements of chromogranin A, chromogranin B (secretogranin I), chromogranin C (secretogranin II) and pancreastatin in plasma and urine from patients with carcinoid tumours and endocrine pancreatic tumours. J Endocrinol 144: 49-59

Strub JM, Goumon Y, Lugardon K, Capon C, Lopez M, Moniatte M, Van Dorsselaer A, Aunis D and Metz-Boutigue MH (1996) Antibacterial activity of glycosylated and phosphorylated chromogranin A-derived peptide 173-194 from bovine adrenal medullary chromaffin granules. J Biol Chem $\mathbf{2 7 1}$ : 28533-28540

Strub JM, Sorokine O, Van Dorsselaer A, Aunis D and Metz-Boutigue MH (1997) Phosphorylation and O-glycosylation sites of bovine chromogranin A from adrenal medullary chromaffin granules and their relationship with biological activities. J Biol Chem 272: 11928-11936

Syversen U, Jacobsen MB, O'Connor DT, Ronning K and Waldum HL (1994) Immunoassays for measurement of chromogranin A and pancreastatin-like immunoreactivity in humans: correspondence in patients with neuroendocrine neoplasia. Neuropeptides 26: 201-206

Taupenot L, Remacle JE, Helle KB, Aunis D and Bader MF (1995). Recombinant human chromogranin A: expression, purification and characterization of the N-terminal derived peptides. Regul Pept 56: 71-88

Watkinson A, Jonsson AC, Davison M, Young J, Lee CM, Moore S and Dockray GJ (1991) Heterogeneity of chromogranin A-derived peptides in bovine gut, pancreas and adrenal medulla. Biochem J 276: 471-479 